\title{
FAMILIES OF DIRAC OPERATORS AND QUANTUM AFFINE GROUPS

\author{
JOUKO MICKELSSON
}

(Received 29 July 2010; accepted 29 December 2010)

Communicated by S. Rosenberg

Dedicated to Alan Carey, on the occasion of his 60th birthday

\begin{abstract}
Twisted $K$-theory classes over compact Lie groups can be realized as families of Fredholm operators using the representation theory of loop groups. In this paper we show how to deform the Fredholm family in the sense of quantum groups. The family of Dirac-type operators is parametrized by vectors in the adjoint module for a quantum affine algebra and transforms covariantly under a central extension of the algebra.
\end{abstract}

2010 Mathematics subject classification: primary 81R10; secondary 17B37, 19L50.

Keywords and phrases: Dirac operator, quantum affine algebra, $K$-theory.

\section{Introduction}

Let $X$ be a topological space and $\mathrm{Fred}_{*}$ the space of self-adjoint Fredholm operators in a complex Hilbert space $H$ with both positive and negative essential spectrum. This is a universal classifying space for $K^{1}$. Indeed, one can take as the definition:

$$
K^{1}(X)=\left\{\text { homotopy classes of maps } f: X \rightarrow \text { Fred }_{*}\right\}
$$

and in the even case

$$
K^{0}(X)=\{\text { homotopy classes of maps } f: X \rightarrow \text { Fred }\},
$$

where Fred is the space of all Fredholm operators in $H$.

The Chern character

$$
\operatorname{ch}: K^{1}(X) \rightarrow H^{\text {odd }}(X, \mathbb{Z})
$$

is an additive map into the set of all odd cohomology classes. In particular, the degreethree component $D D(f)=\operatorname{ch}_{3}(f)$ of $[f] \in K^{1}(X)$ is called the Dixmier-Douady class of the gerbe defined by the family $f(x)$ of Fredholm operators. In the de Rham 
cohomology an equivalent construction of $D D(f)$ comes from the family $L_{\lambda \lambda^{\prime}}$ of complex line bundles. One can choose the curvature forms $\omega_{\lambda \lambda^{\prime}}$ such that

$$
\omega_{\lambda \lambda^{\prime}}+\omega_{\lambda^{\prime} \lambda^{\prime \prime}}=\omega_{\lambda \lambda^{\prime \prime}}
$$

and, with a partition of unity $\sum \rho_{\lambda}=1$ subordinate to the cover by the open sets $U_{\lambda}$, one has

$$
D D(f)=\sum_{\lambda} d \rho_{\lambda} \wedge \omega_{\lambda \lambda^{\prime}}
$$

This does not depend on the choice of $\lambda^{\prime}$. The 3-cohomology class is related to quantum field theory anomalies and it transgresses in the Hamiltonian formulation of gauge theory to central or, more generally, abelian extensions of the group of gauge transformations [2].

A 3-cohomology class $[\mathcal{H}$ ] can be used as a twisting in $K$-theory. Instead of a globally defined family of Fredholm operators over $X$, we have local families $f_{\alpha}: U_{\alpha} \rightarrow \operatorname{Fred}(H)$, where $\left\{U_{\alpha}\right\}$ is an open cover of $X$ that satisfies the condition that on the overlaps $U_{\alpha} \cap U_{\beta}$ we have

$$
f_{\beta}(x)=h_{\beta \alpha}(x) f_{\alpha}(x) h_{\beta \alpha}(x)^{-1} .
$$

Here the $h_{\alpha \beta}$ are the transition functions for a principal $P U(H)$ bundle $P$ over $X$. Recall that $P U(H)=U(H) / S^{1}$ is the group of projective unitaries in $H$. The equivalence class of the bundle $P$ is given by $[\mathcal{H}]$. The local 2-forms $\omega_{\alpha \beta}$ are given as the pullbacks $h_{\alpha \beta}^{*} \omega$, where $\omega$ is the Chern class of the canonical complex line bundle over $P U(H)$.

Quantum field theory provides a method for constructing twisted families of Fredholm operators. In particular, one can use the supersymmetric Wess-ZuminoWitten (WZW) model in the case when $X$ is a compact Lie group. This construction actually provides an operator theoretic realization for the relationship between twisted $K$-theory and the Verlinde algebra in conformal field theory, shown in [4]. In the next section, we will briefly recall the construction of the nondeformed family of Dirac operators [8].

\section{The supersymmetric WZW model}

Families of Dirac operators $D_{A}$ on the unit circle $S^{1}$ coupled to smooth vector potentials $A$ transform covariantly under the gauge group action and so define an element of $K^{*}(\mathcal{A} / \mathcal{G})=K^{*}(G)$. Here $\mathcal{G}=\Omega G$ is the based smooth loop group of a compact Lie group $G$. The quantized Dirac operators $\hat{D}_{A}$ acting in a fermionic Fock space transform covariantly under a central extension $\widehat{L G}$ of the full loop group $L G$. Thus, the family $\hat{D}_{A}$ is a candidate for an element of twisted $K$-theory on the moduli space of gauge connections. However, there is a catch: these operators are essentially positive. We need operators with both positive and negative essential spectrum.

To find genuine nontrivial twisted $K$-theory classes, we have to go to the supersymmetric WZW model. Morally, the family of Fredholm operators is now a 
family of Dirac operators on the loop group $L G$. We cannot make sense of the Dirac operators on the infinite-dimensional manifold $L G$ by standard analytical methods (we would need a Haar measure on the group $L G$ ), but there is a purely algebraic construction using the highest weight representation theory of $\widehat{L G}$.

Concretely, the operators are acting in a tensor product $H=H_{f} \otimes H_{b}$, where $H_{b}$ carries irreducible highest weight representations of $\widehat{L G}$ of level $k=0,1,2, \ldots$ and $H_{f}$ carries an irreducible representation of the Clifford algebra of the vector space $L \mathfrak{g}$. The Clifford algebra is then used to construct a (reducible) representation of the central extension $\hat{\mathfrak{g}}$ of $L \mathfrak{g}$, of level $\kappa$, the dual Coxeter number of $\mathfrak{g}$. A basis for the Clifford algebra consists of elements $\psi_{a}^{n}$ with $n \in \mathbb{Z}$ and $a=1,2, \ldots$, dim $\mathfrak{g}$ with anticommutation relations

$$
\psi_{a}^{n} \psi_{b}^{m}+\psi_{b}^{m} \psi_{a}^{n}=2 \delta_{a b} \delta_{n,-m} .
$$

The commutation relations of the basis vectors $T_{a}^{n}$ of $L \mathfrak{g}$ in the representation space $H_{b}$ are

$$
\left[T_{a}^{n}, T_{b}^{m}\right]=\lambda_{a b c} T_{c}^{n+m}+\frac{k}{4} \delta_{a b} n \delta_{n,-m},
$$

where the $\lambda_{a b c}$ are the structure constants of $\mathfrak{g}$ in a suitably normalized basis and the Dirac operator $Q_{A}$ coupled to a vector potential on $S^{1}$ is

$$
\begin{aligned}
Q_{A} & =i \psi_{a}^{n} \otimes T_{a}^{-n}-\frac{i}{12} \lambda_{a b c} \psi_{a}^{n} \psi_{b}^{m} \psi_{c}^{-n-m} \otimes 1+i \frac{k+\kappa}{4} \psi_{a}^{n} A_{a}^{-n} \otimes 1 \\
& =i \psi_{a}^{n} \otimes T_{a}^{-n}+\frac{i}{3} \psi_{a}^{n} K_{a}^{-n} \otimes 1+i \frac{k+\kappa}{4} \psi_{a}^{n} A_{a}^{-n} \otimes 1 .
\end{aligned}
$$

Here the $K_{a}^{n}$ satisfy the same commutation relations, as operators in $H_{f}$, as the operators $T_{a}^{n}$ except that the level is $\kappa$ instead of $k$. In addition, the $A_{a}^{n}$ are the Fourier components of a vector potential on the circle.

The family $Q_{A}$ transforms the loop group $L G$ covariantly under the projective representation of level $k+\kappa$ defining an element in $K(G, k+\kappa)$ corresponding to the Dixmier-Douady class $[\mathcal{H}]$ in $H^{3}(G, \mathbb{Z})$ equal to $k+\kappa$ times the basic class in $H^{3}(G)=\mathbb{Z}$ when $G$ is a simple, simply connected, compact Lie group.

Indeed, since $\mathcal{A} / \Omega G=G$ and $G \subset L G$, we have a $G$ equivariant class, which is an element of $K_{G}^{*}(G, H)$. In the $q$-deformed case the gauge symmetry is not a group but a Hopf algebra and the moduli space of gauge connections is not defined. Instead, the role of the Dixmier-Douady class $[\mathcal{H}]$ is completely taken over by the (level of) the central extension of the quantum affine algebra.

Our construction can be viewed as a generalization of [1], from the quantum SU(2) algebra to quantum affine algebras. A more detailed article is in preparation [5]. We want to stress that we are not trying to construct a spectral triple, in the sense of noncommutative geometry, using the generalized Dirac operator. Such constructions for compact quantum groups have been discussed in several papers during the last ten years. We refer the interested reader to the recent paper [9] for the references to the earlier literature contained therein. 


\section{Quantum affine algebra}

Let $\mathfrak{g}$ be a simple finite-dimensional Lie algebra and $\hat{\mathfrak{g}}$ the associated affine Lie algebra. The quantum affine algebra $U_{q}(\hat{\mathrm{g}})$ is generated by vectors $e_{0}, e_{1}, \ldots, e_{\ell}$, $f_{0}, f_{1}, \ldots, f_{\ell}$ and $K_{0}, K_{1}, \ldots, K_{\ell}, K_{0}^{-1}, \ldots, K_{\ell}^{-1}$ with the relations

$$
\begin{gathered}
{\left[e_{i}, f_{i}\right]=\delta_{i j} \frac{K_{i}-K_{i}^{-1}}{q-q^{-1}}, \quad K_{i} K_{j}=K_{j} K_{i},} \\
K_{i} e_{j} K_{i}^{-1}=q^{\alpha_{i j}} e_{j}, \quad K_{i} f_{j} K_{i}^{-1}=q^{-\alpha_{i j}} f_{j}, \\
\sum_{k=0}^{1-a_{i j}}(-1)^{k}\left[\begin{array}{c}
1-a_{i j} \\
k
\end{array}\right]_{q} e_{i}^{1-a_{i j}-k} e_{j} e_{i}^{k}=0 \quad(i \neq j), \\
\sum_{k=0}^{1-a_{i j}}(-1)^{k}\left[\begin{array}{c}
1-a_{i j} \\
k
\end{array}\right]_{q} f_{i}^{1-a_{i j}-k} f_{j} f_{i}^{k}=0 \quad(i \neq j),
\end{gathered}
$$

where

$$
\begin{gathered}
{\left[\begin{array}{c}
m \\
k
\end{array}\right]_{q}=\frac{m_{q}(m-1)_{q} \cdots(m-k+1)_{q}}{k_{q}(k-1)_{q} \cdots 1_{q}},} \\
k_{q}=1+q+\cdots+q^{k-1} .
\end{gathered}
$$

Here the parameter $q$ is a positive real number and the integers $a_{i j}$ are the matrix elements of the Cartan matrix of $\hat{\mathfrak{g}}$.

Let $A_{i}^{n}$, for $n \in \mathbb{Z}$ and $i$ a label for the basis in the adjoint module of $\mathfrak{g}$, be a basis for the $q$-affine adjoint module. Under $g$ each 'Fourier mode' $A^{n}$ transforms according to the adjoint representation of $U_{q}(\mathfrak{g})$, which is a $q$-deformation of the adjoint representation of $\mathfrak{g}$. The generator $e_{0}$ increases the index $n$ by one unit, while $f_{0}$ decreases it by one unit. For example, for $\mathfrak{g}=\mathfrak{s l}(2)$ one has the explicit formulas

$$
\begin{aligned}
e_{1} A_{1}^{n} & =f_{0} A_{1}^{n}=0, \quad f_{1} A_{1}^{n}=A_{0}^{n}, \quad e_{0} A_{1}^{n}=A_{0}^{n+1}, \\
e_{1} A_{0}^{n} & =\left(q+q^{-1}\right) A_{1}^{n}, \quad f_{0} A_{0}^{n}=\left(q+q^{-1}\right) A_{1}^{n-1}, \\
f_{1} A_{0}^{n} & =A_{-1}^{n}, \quad e_{0} A_{0}^{n}=A_{-1}^{n+1}, \\
e_{1} A_{-1}^{n} & =\left(q+q^{-1}\right) A_{0}^{n}, \quad f_{0} A_{-1}^{n}=\left(q+q^{-1}\right) A_{0}^{n-1}, \\
f_{1} A_{-1}^{n} & =0=e_{0} A_{-1}^{n}, \\
K_{1} A_{i}^{n} & =q^{2 i} A_{i}^{n}=K_{0}^{-1} A_{i}^{n} .
\end{aligned}
$$

The vectors $A_{i}^{n}$ will be constructed as operators acting in a Fock space carrying a representation of $U_{q}(\hat{\mathfrak{g}})$ such that the adjoint action is given by

$$
x \cdot A_{i}^{n}=\sum_{(x)} x^{\prime} A_{i}^{n} S\left(x^{\prime \prime}\right) \quad \text { for } x \in U_{q}(\hat{\mathfrak{g}}),
$$

where $S: U_{q}(\hat{\mathfrak{g}}) \rightarrow U_{q}(\hat{\mathfrak{g}})$ is the antipode and $\Delta(x)=\sum_{(x)} x^{\prime} \otimes x^{\prime \prime}$ is the coproduct $\Delta: U_{q} \rightarrow U_{q} \otimes U_{q}$. We also need the Clifford algebra generated by elements $\psi_{i}^{n}$ acting in the Fock space and transforming under $U_{q}(\hat{\mathrm{g}})$ according to the dual adjoint representation (which, in fact, is equivalent to the adjoint representation). 


\section{The Dirac operator}

The Dirac operator $Q$ acts on $H_{f} \otimes H_{b}$, where $H_{f}$ is the $q$-fermionic Fock space and $H_{b}$ carries another highest weight representation of $U_{q}(\hat{\mathrm{g}})$. The action of the nontrivial central extension is seen in the action of the element $K_{0} K_{1} \cdots K_{\ell}$, which is no longer equal to the unit operator, but a power of $q$ whose exponent depends on the level of the representation.

$$
Q=i \sum \psi_{a}^{n} \otimes T_{a}^{-n}+i \frac{1}{3} \sum \psi_{a}^{n} K_{a}^{-n} \otimes 1
$$

Here the $T_{a}^{n}$ are basis vectors of the adjoint module, acting as linear operators in the space $H_{b}$. We also need another copy of the adjoint module, acting on the space $H_{f}$. The components are denoted by $K_{a}^{n}$. The vectors $\psi_{a}^{n}$ are elements of a quantum Clifford algebra which act as operators on a $q$-Fock space $H_{f}$. These will be defined in Section 4.1. In contrast to the undeformed case, the operators $K_{a}^{n}, T_{a}^{n}$ do not satisfy the defining relations of the algebra $U_{q}(\hat{\mathrm{g}})$.

Let $R$ be the universal $R$-matrix for the algebra $U_{q}$. An explicit construction is given in [6]. Following [3], we can then define a basis for vectors in a submodule $A \subset U_{q}$ which transform according to an adjoint representation

$$
\operatorname{ad}_{q}(x) v=\sum_{(x)} x^{\prime} v S\left(x^{\prime \prime}\right)
$$

of $U_{q}$ on itself. A basis is defined by

$$
A_{i}^{n}=\sum K_{n, i}^{m, \alpha ; p, \beta}\left(\pi_{m, \alpha ; p, \beta} \otimes \mathrm{id}\right) A,
$$

where $A=\left(R^{T} R-1\right) / h$, with $e^{h}=q$ and $R^{T}=\sigma R \sigma$. Here $\sigma$ permutes the factors in the tensor product $U_{q} \otimes U_{q}$ and the $\pi_{m, \alpha ; p, \beta}$ are the matrix elements in the defining representation $V$ of $U_{q}$.

For example, for $\hat{\mathfrak{g}}=\widehat{\mathfrak{s l}}(2)$ the basis in the defining representation is $v_{\alpha}^{n}$, with $n \in \mathbb{Z}$ and $\alpha, \beta= \pm$, and $i=-1,0,1$ in the formula for $A_{i}^{n}$ above. The numerical coefficients $K$ come from the identification of the basis of the adjoint representation as linear combinations of the basis vectors in $V \otimes V$.

The action of the Serre generators in the defining representation is

$$
\begin{aligned}
& e_{1} v_{+}^{n}=f_{0} v_{+}^{n}=0, \quad f_{1} v_{+}^{n}=v_{-}^{n}, \quad e_{0} v_{+}^{n}=v_{-}^{n+1}, \quad e_{0} v_{+}^{n}=v_{-}^{n-1}, \\
& e_{1} v_{-}^{n}=v_{+}^{n}, \quad f_{0} v_{-}^{n}=v_{+}^{n-1}, \quad e_{0} v_{-}^{n}=0=f_{1} v_{-}^{n}, \\
& K_{1} v_{ \pm}^{n}=q^{ \pm 1} v_{ \pm}^{n}=K_{0}^{-1} v_{ \pm}^{n} .
\end{aligned}
$$

4.1. Generalized affine Hecke algebra, the case of $\left.\boldsymbol{U}_{\boldsymbol{q}} \widehat{(\mathfrak{s l}}(2)\right)$. The affine Hecke algebra for $\hat{\mathfrak{g}}$ is defined through the relations coming from the $R$-matrix $\check{\mathrm{R}}=\sigma R$ in the tensor product $V^{0} \otimes V^{0}$. See [7] for the use of the Hecke algebra for constructing Fock space representations of $U_{q}(\widehat{\mathfrak{s l}}(n))$. The matrix satisfies

$$
\left(\check{\mathrm{R}}-q^{-1}\right)(\check{\mathrm{R}}+q)=0,
$$


since $-q$ and $q^{-1}$ are the only eigenvalues of the invertible matrix $\check{\mathrm{R}}$. Denote by $Y_{1}$ the shift operator which sends $v_{i}^{n} \otimes v_{j}^{m}$ to $v_{i}^{n+1} \otimes v_{j}^{m}$ and by $Y_{2}$ the corresponding shift operator acting on the second tensor factor. The matrix $\check{\mathrm{R}}$ acting on $V$ is then defined using the relations

$$
\check{\mathrm{R}} Y_{1}=Y_{2} \check{\mathrm{R}}^{-1}, \quad \check{\mathrm{R}} Y_{2}=Y_{1} \check{\mathrm{R}}+\left(q-q^{-1}\right) Y_{2} .
$$

Indeed, the second relation follows from the first and the minimal polynomial relation.

Now the braiding relations are given by setting the ideal in the tensor algebra of $V$ generated by the elements

$$
\left(q^{-1}+\check{\mathrm{R}}\right)(V \otimes V)
$$

equal to zero. In particular, these have the consequence that any $v_{i}^{n} v_{j}^{m}$ with $n>m$ can be written as a linear combination of vectors $v_{k}^{p} v_{l}^{q}$ with $p+q=n+m$ and $p \leq q$. In the zero mode space $V^{0}$ the meaning of the braiding relations is that they project out the 'symmetric' part of the tensor product $V^{0} \otimes V^{0}$. The three-dimensional representation is the eigenspace of $\check{\mathrm{R}}$ with eigenvalue $q^{-1}$ and the one-dimensional component corresponds to the eigenvalue $-q$.

To complete the construction of the Dirac operator, we also need the generalized Clifford algebra in the coadjoint representation. The algebra is generated by vectors $\psi_{i}^{n}$ with $n \in \mathbb{Z}$ and $i=1,0,-1$. The defining relations are given by braiding relations and an invariant (nonsymmetric) bilinear form. The braiding relations are defined recursively, similarly to the case for $V$ (or $V^{*}$ ) with the difference that the $R$-matrix $\check{\mathrm{R}}$ in the adjoint representation has three rather than two different eigenvalues. These eigenvalues are now $-q^{-2}, q^{2}$ and $q^{-4}$ with multiplicities 3,5 and 1 , respectively. The Fock space representation of the Clifford algebra is defined similarly to the undeformed case: it is generated by a finite-dimensional irreducible spin representation of the zero Fourier mode algebra, with the additional relation that this subspace is annihilated by the elements $\psi_{i}^{n}$ for $n<0$.

Finally, the action of $\left.U_{q} \widehat{(\mathfrak{s l}}(n)\right)$ is defined in the tensor algebra modulo the ideal generated by the elements (4.1) below, over the coadjoint module, using the opposite coproduct $\Delta^{\mathrm{op}}(x)=\sum_{(x)} x^{\prime \prime} \otimes x^{\prime}$.

The negative eigenvalue corresponds again to a three-dimensional 'antisymmetric' representation and the positive eigenvalues to a six-dimensional 'symmetric' representation. The latter contains the one-dimensional trivial representation. The Hecke algebra is replaced by a generalized Hecke algebra,

$$
\begin{gathered}
Y_{1} Y_{2}=Y_{2} Y_{1}, \\
\left(\check{\mathrm{R}}-q^{2}\right)\left(\check{\mathrm{R}}-q^{-4}\right)\left(\check{\mathrm{R}}+q^{-2}\right)=0, \\
\check{\mathrm{R}} Y_{1}=Y_{2} \check{\mathrm{R}}^{-1}, \quad \check{\mathrm{R}} Y_{2}=Y_{i} \check{\mathrm{R}}+\left(q^{2}-q^{-2}\right) Y_{2},
\end{gathered}
$$

where the middle relation is the minimal polynomial of the diagonalizable matrix $\check{\mathrm{R}}$.

The generalized symmetric tensors correspond to positive eigenvalues of $\check{R}$. In the Clifford algebra, symmetrized products are identified as scalars times the unit. That is, 
we fix a $U_{q} \widehat{(\mathfrak{s l}(2))}$ invariant bilinear form $B$ and the Clifford algebra is defined as the tensor algebra over $V$ modulo the ideal generated by

$$
P(u \otimes v)-B(u, v) \cdot 1,
$$

where $P$ is the projection on the positive spectral subspace of $\check{\mathrm{R}}$. In the case when $V$ is the adjoint module for $\left.U_{q} \widehat{(\mathfrak{s l}}(2)\right)$, one can fix $B$ by identifying the first factor $V$ with the dual $V^{*}$ and using the natural pairing $V^{*} \otimes V \rightarrow \mathbb{C}$. Alternatively, one can view $B$ as the projection onto the one-dimensional trivial submodule inside of the 'symmetric module'.

4.2. The family of Dirac operators. In the nondeformed case one has, for an infinitesimal gauge transformation $X \in L \mathfrak{g}$,

$$
[X, Q]=(k+\kappa) \sum(-n) \psi_{i}^{n} X_{i}^{-n}=\frac{k+\kappa}{4}\langle\psi, d X\rangle
$$

and for, a family of operators $Q_{A}=Q+((k+\kappa) / 4) \psi_{i}^{n} A_{i}^{-n}$,

$$
\left[X, Q_{A}\right]=\frac{k+\kappa}{4}\langle\psi,[A, X]+d X\rangle .
$$

In the $q$-deformed case $A$ is to be understood as a vector in the adjoint module extended by $\mathbb{C} c$. The above equation is replaced by

$$
\sum_{(x)} x^{\prime} Q_{A} S\left(x^{\prime \prime}\right)=Q_{x_{c} A},
$$

where $x_{c}$ denotes the adjoint action in the centrally extended module,

$$
x \cdot{ }_{c} A=x \cdot A+\lambda_{x}(A) c .
$$

Here $\lambda_{x}$ is a linear form on the adjoint module which is linear in the argument $x \in U_{q}(\widehat{\mathfrak{s l}}(2))$. It satisfies the cocycle relation

$$
\lambda_{x y}(A)=\lambda_{x}(y \cdot A) \text {. }
$$

Here $c$ is an element of the extended module such that $x \cdot c=0$ for all $\left.x \in U_{q} \widehat{(\mathfrak{s l}}(2)\right)$. It is enough to give a value of $\lambda_{x}$ when $x$ is a Serre generator. The only nonzero forms are $\lambda_{e_{0}}$ and $\lambda_{f_{0}}$. The former is nonzero only for the component $A=A_{1}^{-1}$ and the latter for $A=A_{-1}^{1}$. In the particular case when the adjoint module is acting as operators in a level 1 representation of $\left.U_{q} \widehat{(\mathfrak{s l}}(2)\right)$, we have $\lambda_{e_{0}}\left(A_{1}^{-1}\right)=-q^{-1}$ and $\lambda_{f_{0}}\left(A_{-1}^{1}\right)=q^{-1}$.

\section{References}

[1] P. N. Bibikov and P. P. Kulish, 'Dirac operators on quantum SU(2) group and quantum sphere', J. Math. Sci. (N. Y.) 100 (2000), 2039-2050.

[2] A. L. Carey, J. Mickelsson and M. K. Murray, 'Bundle gerbes applied to quantum field theory', Rev. Math. Phys. 12 (2000), 65-90.

[3] G. W. Delius, M. D. Gould, A. Hüffmann and Y.-Z. Zhang, 'Quantum Lie algebras associated to $U_{q}\left(g l_{n}\right)$ and $U_{q}\left(s l_{n}\right)^{\prime}$, J. Phys. A 29 (1996), 5611-5618.

[4] D. S. Freed, M. J. Hopkins and C. Teleman, 'Twisted equivariant $K$-theory with complex coefficients', J. Topol. 1 (2008), 16-44. 
[5] A. Harju and J. Mickelsson, in preparation.

[6] S. Khoroshkin and V. Tolstoy, 'Twisting of quantized Lie (super)algebras', in: Quantum Groups, Karpacz, 1994 (Polish Scientific Publishers PWN, Warsaw, 1995), pp. 63-84.

[7] B. Leclerc, 'Fock space representations of $U_{q}(\widehat{s l(n)})$ ', Lecture notes, Grenoble 2008, http://cel.archives-ouvertes.fr/docs/00/43/97/41/PDF/LECLERC_IFETE2008.pdf.

[8] J. Mickelsson, 'Gerbes, (twisted) $K$-theory, and the supersymmetric WZW model', in: Infinite Dimensional Groups and Manifolds, IRMA Lectures in Mathematics and Theoretical Physics, 5 (de Gruyter, Berlin, 2004), pp. 93-107.

[9] S. Neshveyev and L. Tuset, 'The Dirac operator on compact quantum groups', J. Reine Angew. Math. 641 (2010), 1-20.

JOUKO MICKELSSON, Department of Mathematics and Statistics, University of Helsinki, Helsinki, Finland

and

Department of Theoretical Physics, Royal Institute of Technology, Stockholm, Sweden

e-mail: jouko@kth.se 